\title{
Correction to: Chimpanzee (Pan troglodytes schweinfurthii) Group Sleep and Pathogen-Vector Avoidance: Experimental Support for the Encounter-Dilution Effect
}

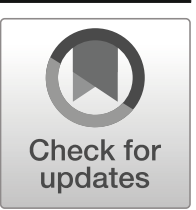

David R. Samson ${ }^{1,2}$ (D) - Luke A. Louden ${ }^{3} \cdot$ Katie Gerstner $^{4} \cdot$ Samantha Wylie $^{5}$. Ben Lake $^{6} \cdot$ Bradley J. White $^{7} \cdot$ Charles L. Nunn ${ }^{2,8} \cdot$ Kevin D. Hunt $^{9}$

Published online: 10 January 2020

(C) Springer Science+Business Media, LLC, part of Springer Nature 2020

\section{Correction to: International Journal of Primatology https://doi.org/10.1007/s10764-019-00111-z}

The original version of this article unfortunately contained a mistake in the authorgroup section. Author Samantha Wylie's family name was incorrectly presented as "Wiley".

The original article has been corrected.

The online version of the original article can be found at https://doi.org/10.1007/s10764-019-00111-z

David R. Samson

david.samson@utoronto.ca

1 Department of Anthropology, University of Toronto, Mississauga, Mississauga, ON, Canada

2 Department of Evolutionary Anthropology, Duke University, Durham, NC, USA

3 Department of Biological Sciences, Purdue University, West Lafayette, IN, USA

4 Department of Anthropology, Wayne State University, Detroit, MI, USA

5 Department of Anthropology, University of Nevada, Las Vegas, NV, USA

6 Lester E. Fisher Center for the Study and Conservation of Apes, Lincoln Park Zoo, Chicago, IL, USA

7 Department of Entomology at the Center for Disease Vector Research, University of California, Riverside, Riverside, CA, USA

8 Duke Global Health Institute, Duke University, Durham, NC, USA

9 Department of Anthropology, Indiana University, Bloomington, IN, USA 